\title{
Tandem duplication of mitochondrial DNA in the black-faced spoonbill, Platalea minor
}

\author{
Hyong-Ju Cho ${ }^{1}$, Masaki Eda ${ }^{2}$, Shin Nishida ${ }^{1}$, Yoshiki Yasukochi ${ }^{1}$, \\ Jong-Ryol Chong ${ }^{3}$ and Hiroko Koike ${ }^{1 *}$ \\ ${ }^{1}$ Laboratory of Biodiversity, Department Environmental Changes, SCS, Kyushu \\ University, 774 Moto-oka, Nishi-ku, Fukuoka City, 819-0395, Japan \\ ${ }^{2}$ Department of Functional Morphology, Faculty of Medicine, \\ Tottori University, Nishimachi 86, Yonago City, \\ Tottori, 683-8503, Japan \\ ${ }^{3}$ Wildlife Research Center, Korea University, 1-700 Ogawacho, \\ Kodaira City, Tokyo, 187-8560, Japan
}

(Received 4 July 2009, accepted 10 October 2009)

\begin{abstract}
Mitochondrial (mt) heteroplasmy in the control region (CR) of the black-faced spoonbill was investigated using LA-PCR. To avoid amplification of transpositioned nuclear genome fragment from mtDNA (numt), PCR product of the almostcomplete mitochondrial genome was amplified using primers designed to anneal on the COIII gene. Then nested LA-PCR product was amplified between the cyt $b$ and $12 S$ rRNA genes using the almost-complete mitochondrial genome PCR product as a template. Nucleotide sequencing revealed tandem duplication composed of two units. The first contains cyt b-1, tRNA ${ }^{\text {Thr }}-1, t R N A^{\text {Pro }}-1, N D 6-1, t R N A^{G l u}-1$ and $\mathrm{CR} 1$, and the second consists of cyt b-2, $t R N A^{\text {Thr }}-2, t R N A^{\text {Pro }}-2, N D 6-2, t R N A^{\text {Glu }}-2$ and CR2, followed by $t R N A^{\text {Phe }}$ and $12 S$ rRNA. The duplicated cyt $b-2$ sequence coincided with $499 \mathrm{bp}$ at the 3 ' end of cyt b-1. With the exception of the CR, the other genes in the duplicated sequence were identical to the original corresponding gene. Even though both CR1 and CR2 contain functional blocks, such as a poly$\mathrm{C}$ site, a goose hairpin and a TAS structure in Domain I, the 3' end of CR1 was followed by a $112 \mathrm{bp}$ sequence (non-coding region) that was not found in CR2 or in sequence homology analysis of similar genes. Meanwhile, CR2 ended in a complicated repeat sequence. The 5' franking region in the Domain I (Region A) and the 3 ' franking region in the Domain I (Region B) of the two CRs evolve in quite different manners: Region A was highly variable between CR1 and CR2 in the same individuals, while Region B was almost identical between them, which indicates concerted evolution.
\end{abstract}

Key words: black-faced spoonbill, concerted evolution, mitochondrial gene order, Platalea minor, tandem duplication

\section{INTRODUCTION}

Control region (CR) of the mitochondrial DNA (mtDNA) is characterized as being the most variable region of mtDNA (Brown et al., 1986; Fauron and Wolstenholme, 1976; Chang and Clayton, 1985), despite its functional significance involved in the initiation of duplication and transcription (Desjardins and Morais, 1990, 1991). Examination of the mutations in this region provides useful information of inter- and intra-species phylogeography (Moore, 1995; Zhang and Hewitt, 1996; Sorenson and

\section{Edited by Hidenori Tachida}

* Corresponding author. E-mail: koikegsc@mbox.nc.kyushu-u.ac.jp
Quinn, 1998).

The avian mitochondrial genome encodes the same set of genes as other vertebrate mtDNAs, and is organized in a very similar fashion. However, they are arranged in a unique gene order. The contiguous $t R N A^{\text {Glu }}$ and ND6 genes are located immediately adjacent to the control region of the molecule, just ahead of the contiguous $t R N A^{P r o}, t R N A^{T h r}$, and cyt $b$ genes (Desjardins and Morais, 1990; Quinn and Wilson, 1993). The avian CR is also divided into a conservative central domain (Domain II) with flanking variable left and right domains (Domains I and III). The high similarity of Domain II observed among members of the avian species is represented by the conservative motives in $\mathrm{C}, \mathrm{D}$, and $\mathrm{F}$ boxes (Brown et al., 
1986), while Domains I and III contain numerous substitutions, indels, and repeat sequences (Wenink et al., 1994; Berg et al., 1995).

PCR techniques have greatly enhanced molecular phylogenetic analysis using mtDNA; however, these techniques have also revealed a high degree of heteroplasmy in mtDNA. The transposition of mtDNA sequence to the nuclear genome (numt) has been documented in a wide variety of taxa from fungi to vertebrates, and mounting evidence suggests that it is a common, if not ubiquitous, phenomenon (Zhang and Hewitt, 1996). Quinn and White (1987) documented the first example of a numt in birds. Numts present interesting opportunities for both the study of molecular evolution and phylogenetic analysis (Zhang and Hewitt, 1996; Quinn, 1997; Sorenson and Quinn, 1998). Arctander (1995) and Sorenson and Fleischer (1996) amplified nuclear sequences from avian blood samples and identified six different numts derived from the mtDNA control region in diving ducks (Aythyini), which are highly similar to the mtDNA sequences of ducks.

Another potential source of apparent heteroplasmy arises from the duplication of a section of mtDNA. Duplicated gene segments, including the CR, have been reported in many taxa, such as ticks (Black and Roehrdanz, 1998; Campbell and Barker, 1999; Shao et al., 2005), an insect (Ogoh and Ohmiya, 2004), sea cucumbers (Arndt and Smith, 1998), fish (Lee et al., 2001), frogs (Sano et al., 2005), lizards (Kumazawa and Endo, 2004; Amer and Kumazawa, 2005), snakes (Kumazawa et al., 1996), and turtles (Parham et al., 2006).

With respect to mtDNA in avian species, falcons and a primary group of songbirds have a unique gene order consisting of $c y t b, t R N A^{T h r}, \mathrm{CR}, t R N A^{\text {Pro }}, N D 6, t R N A^{G l u}$, nc (non-coding sequence, which has similarity with the $\mathrm{CR}$ ), $t R N A^{\text {Phe }}$, and 12S rRNA (Mindell et al., 1998). Parrots of the genus Amazona have duplicated mitochondrial CRs, resulting in a gene order of cyt $b, t R N A^{T h r}, p-N D 6$ (partial $N D 6), p-t R N A^{G l u}$ (partial $t R N A^{G l u}$ ), CR1, $t R N A^{\text {Pro }}, N D 6$, $t R N A^{G l u}$, CR2, $t R N A^{\text {Phe }}$, and 12S rRNA (Eberhard et al., 2001). These CRs contain the typical conserved sequence features.

Albatrosses from the genus Thalassarche (Abbott et al., 2005) also have an altered mtDNA gene order resulting from a duplicated sequence. The duplicated section begins with $d$-cyt $b$ (degenerate partial copy of $c y t b)+$ $p$-cyt $b$ (partial copy of cyt b), followed by $t R N A^{T h r}-2$, $t R N A^{\text {Pro }}-2, N D 6-2, t R N A^{G l u}-2$, and ends with the CR. The $d$-cyt $b$ is a 120 bp fragment with $70 \%$ similarity to $c y t b$, and $p$-cyt $b$ is a $39 \mathrm{bp}$ fragment that is identical to a functional copy of the cyt $b 5$ ' flanking sequence. The total length of CR1 in albatross is $973 \mathrm{bp}$, whereas CR2 is estimated to be up to $2300 \mathrm{bp}$, due to the approximately 1300 bp repeat sequence in Domain III. The degree of similarity between the conserved elements in CR1 and CR2 is thought to be of functional importance. Sequence variations between Region A (110 bp) and B (217 bp) in both CR1 and CR2 are quite dissimilar; Region A sequences in CR1 and CR2 evolved independently, while Region B sequences in both CRs appear to have evolved in concert.

The complete mtDNA genome of the black-faced spoonbill has been reported (GenBank Accession No. NC010962; Lee et al., 2007). We attempted to perform a polymorphic analysis using the CR region of this species, and found that the sequence outputs obtained by direct sequencing contained two peaks which look like heterozygotes. The mtDNA genome recorded in GenBank of this species contained no duplications as the common bird gene order. However, the sequencing of the genome in GenBank was performed using the shotgun method, whereas ours was obtained by direct sequencing of PCR products.

This study describes an investigation into the source of mitochondrial heteroplasmy in the black-faced spoonbill (Hancock et al., 1992; Matheu and Hoyo, 1992). We hypothesized that the most probable cause was a combination of numt (Sorenson and Fleischer, 1996; Kidd and Friesen, 1998; Ruokonen et al., 2000) and secondary heteroplasmy, which gives rise to two types of mtDNA in the cells of an individual (Grzybowski, 2000). A third possibility that we investigated was duplication of the CR region, which has been reported to occur in several avian taxa (Mindell et al., 1998; Abbott et al., 2005; Gibb et al., 2007; Tatarenkov and Avise, 2007).

\section{MATERIALS AND METHODS}

Muscle samples from two individuals (Lab. No. 08kh03 and 08kh04) found dead at the Beppu river mouth in Aira Town, Kagoshima Prefecture, were obtained from the Aira Animal Hospital and stored in 99\% ethanol. Approximately $2 \mathrm{mg}$ of sliced tissue was placed in $285 \mu \mathrm{l}$ of cell lysis solution (Puregen Inc., USA) with $15 \mu \mathrm{l}$ of 20 $\mathrm{mg} / \mathrm{ml}$ proteinase $\mathrm{K}$ and incubated for 2 hours at $55^{\circ} \mathrm{C}$ on a rotator. Nucleic acids were extracted using the Puregen Tissue Kit (Puregen Inc., USA).

To avoid amplification of the numt fragment, the almost-complete mitochondrial PCR product was amplified by the LA-PCR method with a primer set (LCO3.kh and HCO3.kh) designed to anneal to the COIII gene (Fig. 1). Then nested LA-PCR product was amplified between the cyt $b$ and $12 S$ rRNA genes using the almost-complete mitochondrial genome PCR product as a template with TaKaRa LA Taq polymerase (TaKaRa Bio Inc., Otsu, Japan) using a Lcytb1.kh and $12 \mathrm{sR} 2 . \mathrm{kh}$ primer set, of which, the latter was designed based on 12sR2 (Abbott et al., 2005) sequences, and on the mtDNA sequence submitted by Lee et al. (2007; Accession No. NC_010962). Duplication was examined using an Lcon9.kh and Hcon1.kh primer set with TaKaRa Ex Taq HS polymerase (TaKaRa Bio Inc. Otsu, Japan). Five specific internal 
primer sets (Lcytb1.kh and Hnon-c.kh, LND6.kh and Hcyt b4.kh, Lnon-c.kh and H454.gr, Lcon1.kh and Hcon2.kh, LND6.kh and 12sR2.kh) were designed (Table 1) and used for sequencing.

PCR products were enzymatically purified (PCR Product

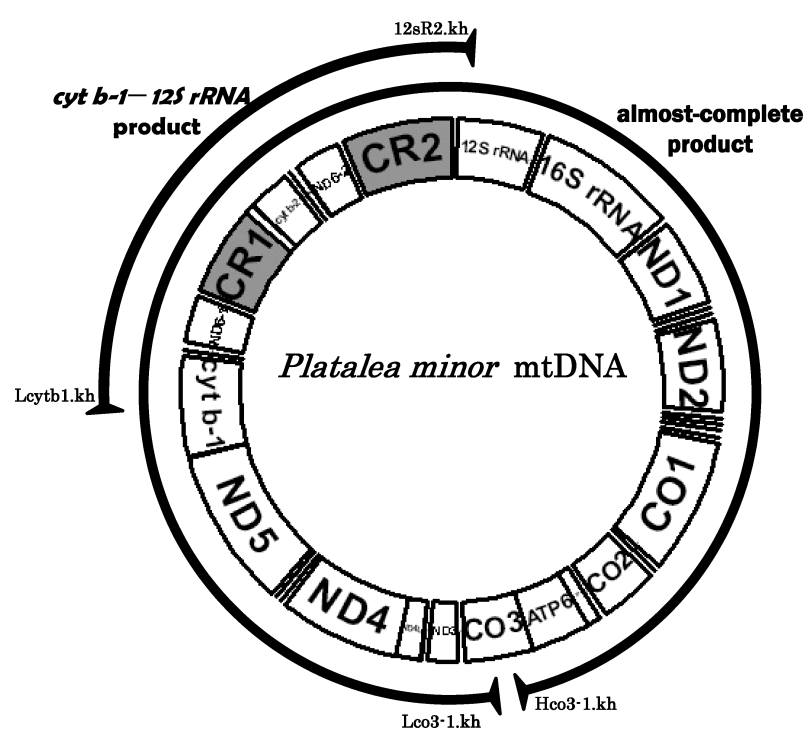

Fig. 1. Schematic representation of the mitochondrial (mt) genome of the black-faced spoonbill, Platalea minor. PCR products of the almost complete $\mathrm{mt}$ genome and sequence determined region (cyt $b$ to $12 S$ rRNA) are shown as arrows.
Pre-Sequencing Kit; USB Corp., USA), and direct sequencing was performed with a Dye Terminator Cycle Sequencing Kit (Beckman Coulter Inc., USA) following the manufacturer's protocol. Cycle sequencing consisted of 30 cycles of $96^{\circ} \mathrm{C}$ for $20 \mathrm{sec}, 50^{\circ} \mathrm{C}$ for $20 \mathrm{sec}$, and $60^{\circ} \mathrm{C}$ for 150 sec. Capillary sequencing was conducted with a CEQ 2000 XL DNA Sequence Analysis System (Beckman Coulter Inc., USA), and the detected waves were analyzed using CEQ Software, Sequence Analysis version 2 (Beckman Coulter Inc., USA). Alignment of sequence data and construction of a neighbor-joining tree were performed using MEGA 4 (Tamura et al., 2007). Genetic distances were calculated using Kimura-2 parameter method (Kimura, 1980) with bootstrap of 1000 replications.

\section{RESULTS}

Gene order LA-PCR products between $c y t b$ and $12 S$ $r R N A$ which were amplified using the almost-complete mitochondrial PCR product as a template (Fig. 1) were determined using five PCR fragments (A, B, C, D and E) which were obtained by amplification using internal primers (Fig. 2). Our results (Accession No. AB519130 to AB519131) show that the gene order of mtDNA in the black-faced spoonbill contains a tandem duplication composed of two units. The first is composed of the sequences for cyt $b-1, t R N A^{T h r}-1, t R N A^{P r o}-1, N D 6-1$,

Table 1. Primer list used in this study

\begin{tabular}{llll}
\hline \hline primer & gene & Sequence 5'-3' & Source \\
\hline Lcytb1.kh & $c y t b^{1}$ & GCTATACACTATACCGCAGACAC & This study \\
cbF1.kh & $c y t b^{1,2}$ & TTGAATCGGCAGCCAACCCGTAG & (Abbott and Double 2003) \\
Hcytb4.kh & $c y t b^{1,2}$ & GATGAAGGGGTGCTCTACGG & This study \\
LND6.kh & $N D 6^{1,2}$ & CCTGCACCATACCCTATAACACGTCAATCC & This study \\
HND6.kh & $N D 6^{1,2}$ & GGATTGACGTGTTATAGGGTATGGTGCAGG & This study \\
Lcon1.kh & $\mathrm{CR}^{1,2}$ & GCCCCATTATATTGCAATAATGCTAGG & This study \\
Lcon4.kh & $\mathrm{CR}^{1}$ & ACAATAATATGTAAGGTGACAATAATCC & This study \\
Lcon5.kh & $\mathrm{CR}^{2}$ & ACAATAACATGTAGGGTGACAACAATCT & This study \\
Lcon6.kh & $\mathrm{CR}^{1,2}$ & ATGAATGCTCGCCTGGCACCCTTGCCACTC & This study \\
Lcon9.kh & $\mathrm{CR}^{1,2}$ & GGGTATGGGGAATCATCTTGACACTG & This study \\
Lcon8.kh & $\mathrm{CR}^{1,2}$ & TTCACATGTCATCAGCACTGGAATTAC & This study \\
Hcon1.kh & $\mathrm{CR}^{1,2}$ & GGGGTCCGTATAGGGGTTATTTATTTTTCG & This study \\
H454.gr & $\mathrm{CR}^{1,2}$ & GCCCTGACATAGGAACCAGAG & Baba et al. 2001 \\
Hcon2.kh & $\mathrm{CR}^{2}$ & GGGACGTTTGTGCACGTGCATGTGAC & This study \\
Lnon-c.kh & non cording region & CTCGAACATCCTCCAAACACCACGACCATC & This study \\
Hnon-c.kh & non cording region & TTGATGGGGATGGATGTGCGATGGGTAAGG & This study \\
12sR2.kh & $12 S r R N A$ & GCTATAACTAAGTCAAGTTTACACTTATTG & (Abbott et al. 2005) \\
Lco3-1.kh & $C O I I$ & TCGGGTCCTCCTTCCTATCAGTCTGCCTCTTAC & This study \\
Hco3-1.kh & $C O I I I$ & TGTTACGGTAACACCTGATGCTAGGAGGATGGC & This study \\
\hline$* 1$ and ${ }^{2}$ in gene indicate whether the primer can amplify both duplicated gene or one of them.
\end{tabular}




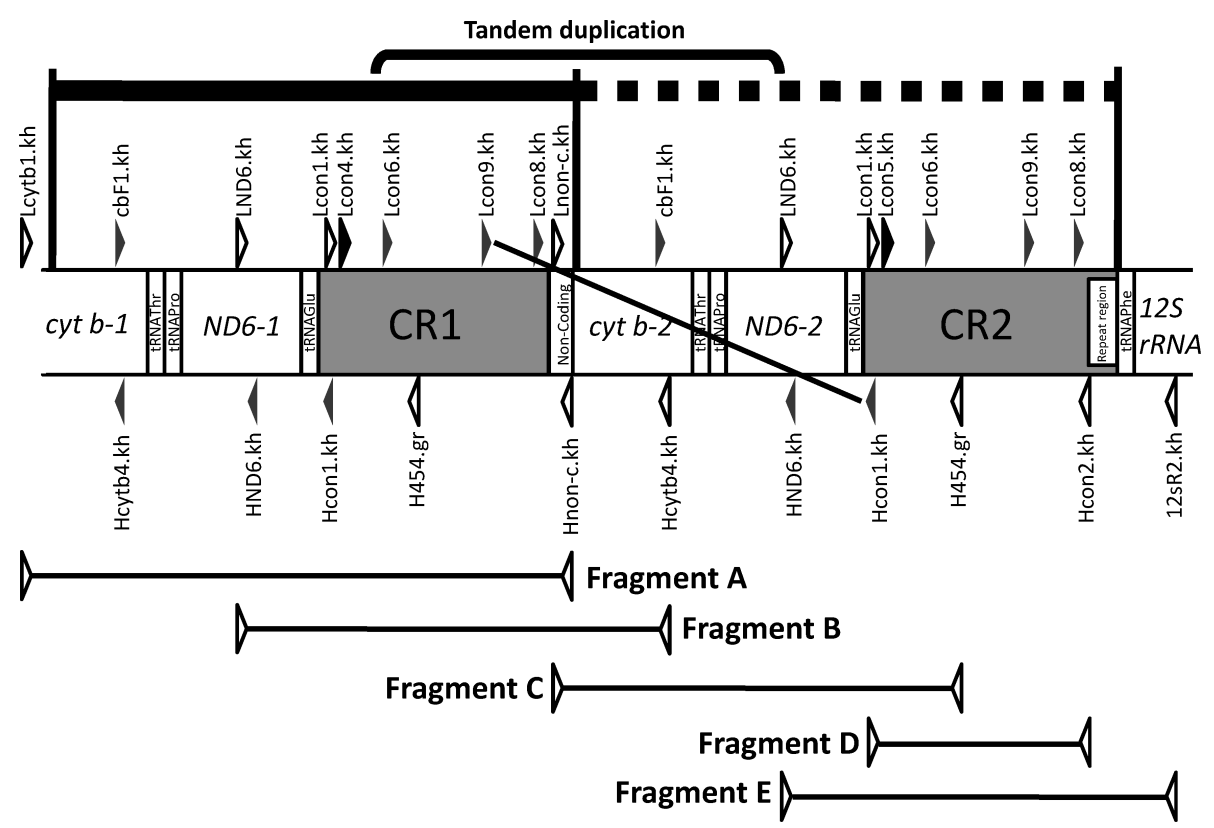

Fig. 2. Schematic representation of mitochondrial gene order and the position of primers for the black-faced spoonbill. Open arrowheads indicate primers used for amplification of fragments. Shaded arrowheads indicate inner-primers used for sequencing. Black arrowheads primers, Lcon4.kh and Lcon5.kh are used to distinguish CR1 and CR2, respectively. Line connecting Lcon9.kh and Hcon1.kh shows a PCR product to indicate the tandem duplication of this area.

$t R N A^{G l u}-1$, and CR1, and the second of $c y t b-2, t R N A^{T h r}-2$, $t R N A^{P r o}-2, N D 6-2, t R N A^{G l u}-2$, and CR2, followed by $t R N A^{\text {Phe }}$ and $12 S$ rRNA.

The presence of the tandem repeat of these genes were also determined by PCR using Lcon9.kh annealed at middle of CR region and Hcon1.kh annealed near 3'end of $\mathrm{CR}$ region. It resulted in $2300 \mathrm{bp}$ of PCR fragment containing 5' franking region of CR1, cyt b-2, tRNAThr-2, $t R N A^{P r o}-2, N D 6-2, t R N A^{G l u}-2$, and 3'end of CR2 (Fig. 2), instead of almost-complete mitochondrial PCR product.

Comparison between these tandem-duplicated genes Sequence analysis determined that the duplicated cyt b-2 sequence coincided with $499 \mathrm{bp}$ at the 5'end of $c y t b-1$. The sequences for $t R N A^{\text {Thr }}-2, t R N A^{P r o}-2$, and $t R N A^{G l u}$-2 were also identical to those for $t R N A^{T h r}-1$, $t R N A^{P r o}-1$, and $t R N A^{G l u}-1$, respectively, and the $N D 6-2$ sequence matched the ND6-1 sequence.

On the other hand, sequencing of the duplicated CRs of this species revealed complicated structures. The 1007 bp at the 3' end of both of the CRs had a high similarity and was readily aligned. Domain I of both CR1 and CR2 was $364 \mathrm{bp}$ in length and had a poly-C site and a TAS element (Ramirez et al., 1993). The region between the 47th bp and 211st bp at the 3' end of Domain I contained one indel and 18 substitutions, which enabled us to discriminate CR1 from CR2. The remaining sequences in Domain I were highly similar, with the exception of two substitutions between CR1 and CR2.
Domain II is the most conserved domain in the control region, and contains the $\mathrm{F}$ box, $\mathrm{D}$ box, $\mathrm{C}$ box, and $\mathrm{BSB}$ (Bird Similarity Box). The Domain II sequences of both CR1 and CR2 had a length of $463 \mathrm{bp}$ and contained these elements. In Domain III, the CSB-1 (ConservedSequence Block-1; Sbisa et al., 1997) was detected in both CR1 and CR2, and the $181 \mathrm{bp}$ at the beginning of Domain III was identical between the two CRs. The 3' end of CR1 is followed by a 112 bp non-coding region, which could not be aligned to CR2, and could not be matched with a similar mtDNA sequence by a BLAST search. The 3 ' end of CR2 contains a relatively long and complicated repeat sequence, beginning at $251 \mathrm{bp}$ from the start of Domain III. This repeat sequence contains two CA-rich repeats measuring $11 \mathrm{bp}$ and $22 \mathrm{bp}$ in length.

Dissimilarity of Domain I in CR1 and CR2 To examine the variation arising from substitutions in CR1 and CR2, an additional set of primers was designed that was able to distinguish CR1 and CR2 (Lcont4.kh and Lcont5.kh; Fig. 2). MtDNA from the two individuals from Kagoshima and from fourteen fallen feathers collected in 2007 from Imazu estuary in Fukuoka city (AB519132-AB519141) were analyzed using the primer sets Lcont4.kh and H454.gr for the partial CR1 region, and Lcont5.kh and H454.gr for the CR2 region. Subsequently, five haplotypes were defined and aligned as shown in Fig. 3.

$\mathrm{CR}$ region sequences by a direct sequencing of PCR 


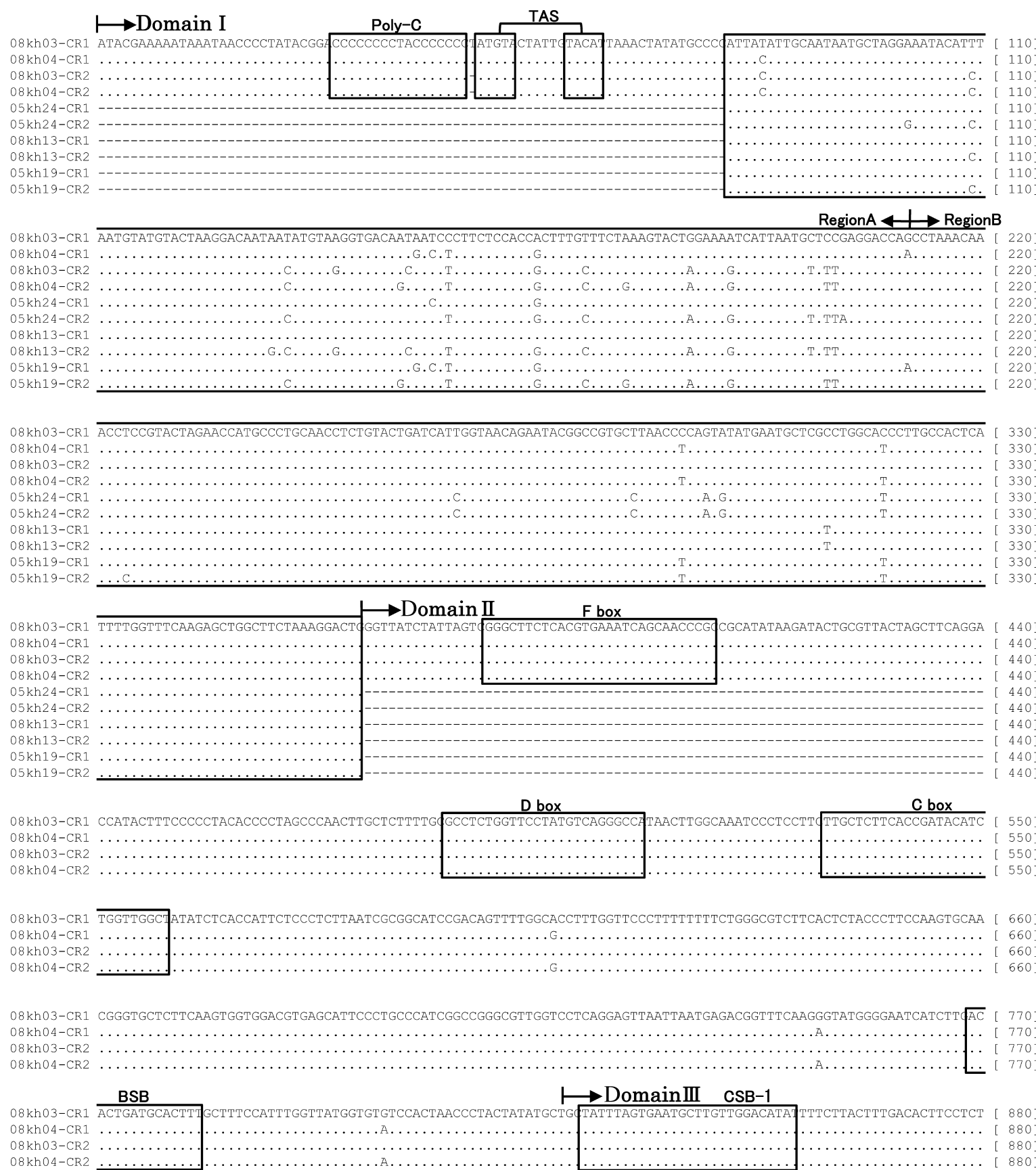

08kh03-CR1 AACTTTCTAAACAACACTAGTAGCTTTTCAGCTAAATATTTGTTGCATTATCGTCATGAATTTTCATGAATTTTTTTTACACTTTTTTCACATGTCATCAGCACTGGAAT [ 990

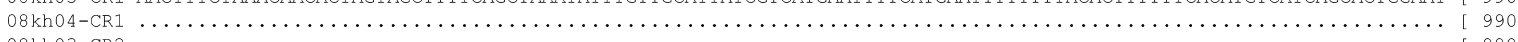

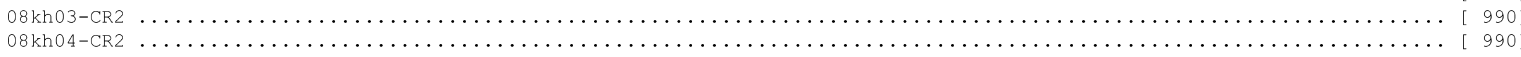

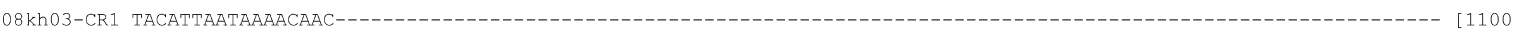

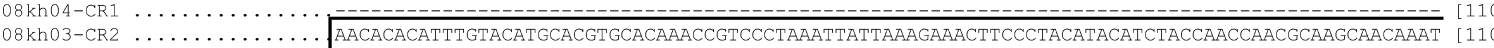

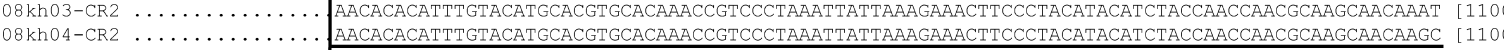
$\rightarrow$ Repert region

$08 \mathrm{kh03-CR}$ $08 \mathrm{kh04- \textrm {CR }}$

8 kh04-CR2 AAGCAACAAGCAAGCAACAAATAAGCAACAAGCAAGCAACAAATAAGCAACAAGCAAGCAACAAA [1165]

CSB : Conserved Sequence Block BSB : Bird Similarity Box

Fig. 3. Alignment of duplicated sequences in the mitochondrial control regions of the black-faced spoonbill. Two control regions (CR1 and CR2) from two individuals are marked to indicate the Poly-C region, Termination Associated Sequence (TAS), F-, D-, B-, and Bird similarity (BSB) boxes, and the Conserved Sequence Block-1 (CSB-1). 

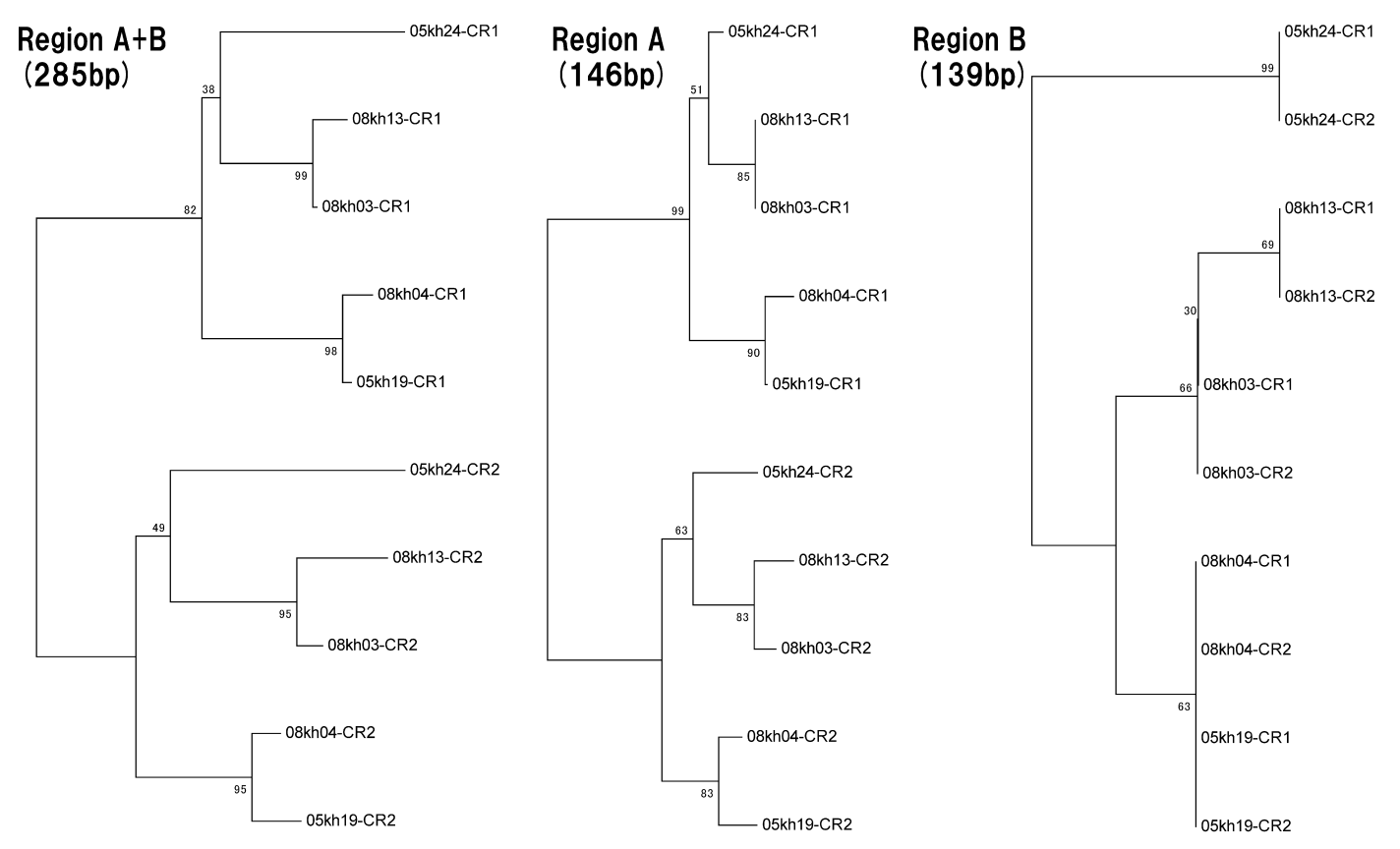

$\longmapsto .005$

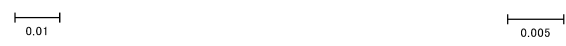

Fig. 4. Neighbor-joining trees constructed using Region A + B (285 bp), Region A (146 bp) and Region B (139 bp) in CR1 and CR2 of 5 individuals.

product amplified with Lcon1.kh which anneals both CR1 and CR2 had many double peaks, especially in Domain I. When CR1 and CR2 were amplified separately using Lcon4.kh annealed at 3'end of CR1 and Lcon5.kh annealed at that of CR2, they did not show any double peaks in their outputs. Compared with CR1 sequence and CR2 sequence, every double peak was interpreted clearly, suggesting that our observation of double peaks, or heteroplasmy of mtDNA must be duplication of CRs.

When CR1 and CR2 were aligned separately, there were clear substitution differences in the first $146 \mathrm{bp}$ of Domain I (Region A) and the final 139 bp of Domain I (Region B). In Region A, most of the substitutions in CR1 and CR2 were different from each other. By contrast, the substitutions in Region B were almost identical between CR1 and CR2 in the same individuals.

Three neighbor-joining (NJ) trees constructed using Region A, Region B and Regions A + B (Fig. 4) revealed a different topology. The NJ trees for Region $\mathrm{A}$ and Regions A + B were divided into 2 clades: CR1 and CR2. The bootstrap value dividing CR1 and CR2 clades in the tree for Region A (99\%) was higher than that of the tree for Region $\mathrm{A}+\mathrm{B}(82 \%)$. On the other hand, in the NJ tree for Region B, CR1 and CR2 from the same individuals were closely clustered.

\section{DISCUSSION}

Avian gene order In this study, tandem duplication of the region between cyt $b$ and the CR was found in the mtDNA of the black-faced spoonbill. The gene order of the tandem duplication of this species begins with cyt $b-1$, $t R N A^{T h r}-1, t R N A^{P r o}-1, N D 6-1, t R N A^{G l u}-1$, and CR1, and is repeated in cyt $b-2, t R N A^{T h r}-2$, tRNA $^{\text {Pro }}-2, N D 6-2, t R N A^{\text {Glu }}-2$, and CR2. This gene order is similar to that of the genus Thalassarche (Abbott et al., 2005), with the exception of $c y t b$-2, which is composed of $d$-cyt $b$ (a 120 bp degenerated copy of $c y t b$ ) and p-cyt $b$ (a copy of the last $39 \mathrm{bp}$ of $c y t b$ ) in the latter.

Gibb et al. (2007) proposed 4 types of avian gene order: Standard avian (chicken), duplicated $t R N A^{T h r}$-CR (albatross), duplicated CR (Amazona parrots), and remnant CR2 (falcon), and indicated that the albatross gene order represents the initial reorganization of avian gene order resulting from gene duplication (Fig. 5). The black-faced spoonbill has longer tandem duplication than any other avian species detected until now, suggesting that the black-faced spoonbill may exhibit an initial type of tandem duplication of mtDNA. Even the black-faced spoonbill has the longest tandem duplication than that of albatross, then parrots and falcon, it is not necessary to indicate evolutional order of Aves, because recent phylogeny of Aves by Hackett et al. (2008) are different from it, suggesting that mtDNA tandem duplication around CR may have occurred independently in several taxa.

Comparison of CR1 and CR2 Ruokonen and Kvist (2002) extensively described the structural and evolution- 

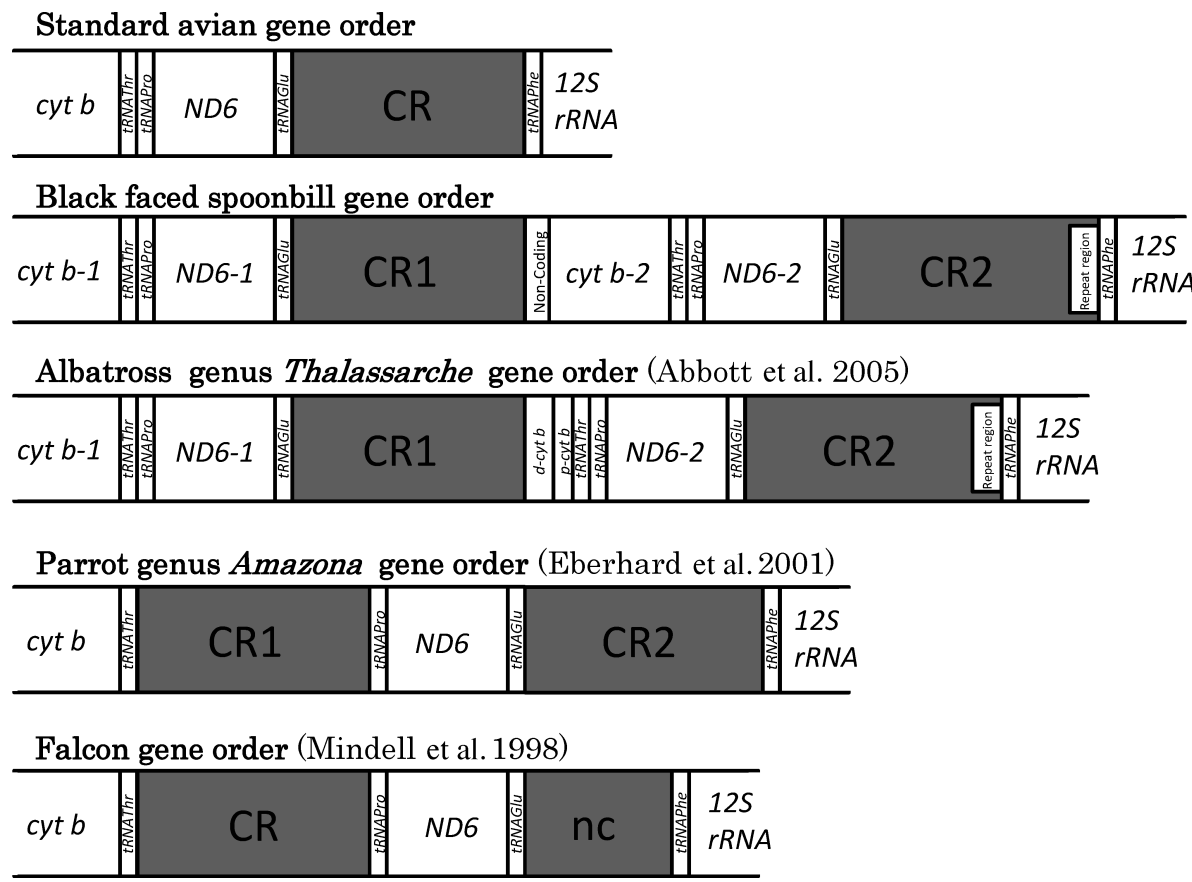

Fig. 5. Schematic representation of mitochondrial gene order in chicken (standard), black-faced spoonbill, albatross, parrot and falcon.

ary characteristics of the CRs of 68 avian species. A poly-C sequence was detected near the 5' end of Domain I in the black-faced spoonbill (Fig. 3), which is a conserved feature in many birds, including Struthioniformes, Galliformes, Falconiformes, and Sphenisciformes (Haring et al., 2001; Ritchie and Lambert, 2000). The conserved palindrome motifs, or TAS (Termination-associated sequence) which consisted of 5'-TACAT-3' (Saccone et al., 1991) and 5'-ATGTA-3' palindrome motif (Desjardins and Morais, 1990) were identified in this species (Fig. 3).

CSB1 (Sbisa et al., 1997), which regulates duplication of the H-strand (Walberg and Clayton, 1981), was identified at the beginning of Domain III, as were other conserved blocks of the F, E, D, and C boxes (Brown et al., 1986), and a BSB (Bird Similarity Box; Ruokonen and Kvist, 2002) in Domain II. Because these features were preserved in both CRs, they may play an important functional role. In addition, CR2 contained a long complicated repeat at the 5' end of Domain III (Piganeau et al., 2004; Mjelle et al., 2008,). At this position, the repeat structure might signal the termination of transcription.

In Domain I, Region A was highly variable between CR1 and CR2 in the same individual, while Region B in CR1 and CR2 was similar. The simultaneous genetic variation observed in Region B is termed "concerted evolution" ( $\mathrm{Li}, 1997$ ), as typified by five species of the albatross mitochondrial genome (Abbott et al., 2005). According to $\mathrm{Li}$ (1997) concerted evolution was first suggested as co-evolution by Edelman and Gally (1970) in the rDNA from Xenopus (Brown et al., 1972). Concerted evolution is now commonly used in a high interspecific homogeneity of the multigene families (Zimmer et al., 1980). Mechanism of concerted evolution of tandemlyrepeated gene such as rDNA is explained in Liao (2000).

The discovery of these tandem repeats in mtDNA in several species in the same genus (Eberhard et al., 2001; Abbott et al., 2005) indicates that we should further investigate the species in genus Plataea or family Threskiornithidae (Orbis group).

\section{REFERENCES}

Abbott, C. L., and Double, M. C. (2003) Phylogeography of shy and white-capped albatrosses inferred from mitochondrial DNA sequences: implications for population history and taxonomy. Mol. Ecol. 12, 2747-2758.

Abbott, C. L., Double, M. C., Trueman, J. W. H. A., Robinson, A., and Cockburn, A. (2005) An unusual source of apparent mitochondrial heteroplasmy: duplicate mitochondrial control regions in Thalassarche albatrosses. Mol. Ecol. 14, 3605-3613.

Amer, S. A. M., and Kumazawa, Y. (2005) Mitochondrial genome of Pogona vitticepes (Reptilia; Agamidae): control region duplication and the origin of Australasian agamids. Gene 346, 249-256.

Arctander, P. (1995) Comparison of a mitochondrial gene and a corresponding nuclear pseudogene. Proc. Biol. Sci. 262, $13-19$.

Arndt, A., and Smith, M. J. (1998) Mitochondrial gene rearrangement in the sea cucumber genus Cucumaria. Mol. Biol. Evol. 15, 1009-1016.

Baba, Y., Fujimaki, Y., Yoshii, R., and Koike, H. (2001) Genetic variability in the mitochondrial control region of the 
Japanese rock ptarmigan Lagopus mutus japonicus. Jpn. J. Ornithol. 56, 53-64.

Berg, T., Moum, T., and Johansen, S. (1995) Variable numbers of tandem repeats make birds of the order Ciconiiformes heteroplasmic in their mitochondrial genomes. Cur. Genet. 27, 257-262.

Black, W. C. IV, and Roehrdanz, R. L. (1998) Mitochondrial gene order is not conserved in arthropods: Prostriate and metastriate tick mitochondrial genomes. Mol. Biol. Evol. 15, 1772-1785.

Brown, D. D., Wensink, P. C., and Jordan, E. (1972) A comparison of the ribosomal DNAs of Xenopus laevis and Xenopus mulleri: Evolution of tandem genes. J. Mol. Biol. 63, 5773.

Brown, G. G., Gadaleta, G., Pepe, G., Saccone, C., and Sbisa, E. (1986) Structural conservation and variation in the D loopcontaining region of vertebrate mitochondrial DNA. J. Mol. Biol. 192, 503-511.

Campbell, N. J. H., and Barker, S. C. (1999) The novel mitochondrial gene arrangement of the cattle tick, Boophilus microplus: Fivefold tandem repetition of a coding region. Mol. Biol. Evol. 16, 732-740.

Chang, D. D., and Clayton, D. A. (1985) Printing for human mitochondrial DNA replication occurs at the light-strand promoter. Proc. Natl. Acad. Sci. USA 82, 351-355.

Desjardins, P., and Morais, R. (1990) Sequence and gene organization of the Chicken mitochondrial genome. J. Mol. Biol. 212, 599-634.

Desjardins, P., and Morais, R. (1991) Nucleotide sequence and evolution of coding and noncoding regions of a quail mitochondrial genome. J. Mol. Evol. 32, 153-161.

Eberhard, J. R., Wright, T. F., and Bermingham, E. (2001) Duplication and concerted evolution of the mitochondrial control region in the parrot genus Amazona. Mol. Biol. Evol. 18, $1330-1342$.

Edelman, G. M., and Gally, J. A. (1970) Arrangement and evolution of eukaryotic genes. In: The Neurosciences: Second Study Program (ed: Schmitt, F.O.), pp. 962-972. Rockfeller University Press, New York.

Fauron, C. M.-R., and Wolstenholme, D. R. (1976) Structural heterogeneity of mitochondrial DNA molecules within the genus Drosophila. Proc. Natl. Acad. Sci. USA 73, 36233627.

Gibb, G. C., Kardailsky, O., Kimball, R. T., Braun, E. L., and Penny, D. (2007) Mitochondrial genomes and avian phylogeny: complex characters and resolvability without explosive radiations. Mol. Biol. Evol. 24, 269-280.

Grzybowski, T. (2000) Extremely high levels of human mitochondrial DNA heteroplasmy in single hair roots. Electrophoresis 21, 548-553.

Hackett, S. J., Kimball, R. T., Reddy, S., Bowie, R. C. K., Braun, E. L., Braun, M. J., Chojnowski, J. L., Cox, W. A., Han, K.-L., Harshman, J., et al. (2008) A phylogenomic study of birds reveals their evolutionary history. Science 320, 1763-1768.

Hancock, J. A., Kushlan, J., and Kahl, M. P. (1992) Storks, Ibises, and Spoonbills of the World, Academic Press, San Diego.

Haring, E., Kruckenhauser, L., Gamauf, A., Riesing, M. J., and Pinsker, W. (2001) The complete sequence of the mitochondrial genome of Buteo buteo Aves, Accipitridae indicates an early split in the phylogeny of raptors. Mol. Biol. Evol. 18, 1892-1904.

Kidd, M. G., and Friesen, V. L. (1998) Sequence variation in the guillemot (Alcidae: Cepphus) mitochondrial control region and its nuclear homolog. Mol. Biol. Evol. 15, 61-70.
Kimura, M. (1980) A simple method for estimating evolutionary rates of base substitutions through comparative studies of nucleotide sequences. J. Mol. Evol. 16, 111-120.

Kumazawa, Y., and Endo, H. (2004) Mitochondrial genome of the Komodo dragon : Efficient sequencing method with reptile-oriented primers and novel gene rearrangements. DNA Res. 11, 115-125.

Kumazawa, Y., Ota, H., Nishida, M., and Ozawa, T. (1996) Gene rearrangements in snake mitochondrial genomes: Highly concerted evolution of control-region-like sequences duplicated and inserted into a tRNA gene cluster. Mol. Biol. Evol. 13, 1242-1254.

Lee, J. S., Miya, M., Lee, Y. S., Kim, C. G., Park, E. H., Aoki, Y., and Nishida, M. (2001) The complete DNA sequence of the mitochondrial genome of the selffertilizing fish Rivulus marmoratus (Cyprinodontiformes, Rivulidae) and the first description of duplication of a control region in fish. Gene 280, 1-7.

Lee,Y. I., Tsai, C-L., Fang, S. Y., and Chou, Y. C. (2007) The complete sequence of Platalea minor mitochondrial genome. NCBI Reference Sequence: NC_010962.1.

Li, W.-H. (1997) Concerted evolution of multigene families. In: Molecular Evolution (ed.: W.-H. Li), pp. 309-334. Sinauer Association Inc. Publishers, Sunderland, MA.

Liao, D. (2000) Gene conversion drives within genic sequences: concerted evolution of ribosomal RNA genes in bacteria and archaea. J. Mol. Evol. 51, 305-317.

Matheu, E., and del Hoyo, J. (1992) Family Threskiornithidae (Ibises and Spoonbills). In: Handbook of the birds of the world (eds.: J. del Hoyo, A. Elliott, and J. Sargatal), Vol. 1, pp. 472-507. Lynx Edicions, New York.

Mindell, D. P., Sorenson, M. D., and Dimcheff, D. E. (1998) Multiple independent origins of mitochondrial gene order in birds. Proc. Natl. Acad. Sci. USA 95, 10693-10697.

Mjelle, K. A., Karlson, B. O., Jorgensen, T. E., Moum, T., and Johansen, S. D. (2008) Halibut mitochondrial genomes contain excessive heteroplasmic tandem repeat arrays involved in DNA recombination. BMC Genomics 9, 10-21.

Moore, W. S. (1995) Inferring phylogenies from mtDNA variation: Mitochondrial-gene trees versus nuclear-gene trees. Evolution 49, 718-726.

Ogoh, K., and Ohmiya, Y. (2004) Complete mitochondrial DNA sequence of the seq-firefly, Vargula hilgendorfii (Crustaea, Ostracoda) with duplicate control regions. Gene 327, 131139.

Parham, J. F., Feldman, C. R., and Boore, J. L. (2006) The complete mitochondrial genome of the enigmatic bigheaded turtle (Platysternon): description of unusual genomic features and the reconciliation of phylogenetic hypotheses based on mitochondrial and nuclear DNA. BMC Evol. Biol. 6, $1-11$

Piganeau, G., Gardner, M., and Eyre-Walker, A. (2004) A broad survey of recombination in animal mitochondria. Mol. Biol. Evol. 21, 2319-2325.

Quinn, T. W. (1997) Molecular evolution of the avian mitochondrial genome. In: Avian Evolution and Molecular Characters (ed.: D.P. Mindell), pp. 3-28. Academic Press, Orlando.

Quinn, T. W., and White, B. N. (1987) Analysis of DNA sequence variation. In: Avian genetics (eds.: F. Cooke and P. A. Buckley), pp. 163-198. Academic Press, London.

Quinn, T. W., and Wilson, A. C., (1993) Sequence evolution in and around the mitochondrial control fregion in birds. J. Mol. Evol. 37, 417-425.

Ramirez, V., Savoie, P., and Morais, R. (1993) Molecular characterization and evolution of a duck mitochondrial genome. 
J. Mol. Evol. 37, 296-310.

Ritchie, P., and Lambert, D. (2000) A repeat complex in the mitochondrial control region of Adélie penguins from Antarctica. Genome 43, 613-618.

Ruokonen, M., Kvist, L., and Lumme, J. (2000) Close relatedness between mitochondrial DNA from seven Anser goose species. J. Evol. Biol. 13, 532-540.

Ruokonen, M., and Kvist, L. (2002) Structure and evolution of the avian mitochondrial control region. Mol. Phylogenet. Evol. 23, 422-432.

Saccone, C., Pesole, G., and Sbisá, E. (1991) The main regulatory region of mammalian mitochondrial DNA: structurefunction model and evolutionary pattern. J. Mol. Evol. 33, 83-91.

Sano, N., Kurabayashi, A., Fujii, T., Yonekawa, H., and Sumida, M. (2005) Complete nucleotide sequence of the mitochondrial genome of Schlegel's tree frog Rhacophorus schlegelii (family Rhacophoridae): duplicated control regions and gene rearrangements. Genes Genet. Syst. 80, 213-224.

Sbisa, E., Tanzariello, F., Reyes, A., Pesole, G., and Saccone, C. (1997) Mammalian mitochondrial D-loop region structural analysis: identification of new conserved sequences and their functional and evolutionary implications. Gene 205, $125-140$

Shao, R., Barker, S. C., Mitani, H., Aoki, Y., and Fukunaga, M. (2005) Evolution of duplicate control regions in the mitochondrial genomes of metazoa: A case study with Australasian Ixodes ticks. Mol. Biol. Evol. 22, 620-629.
Sorenson, M. D., and Fleischer, R. C. (1996) Multiple independent transpositions of mitochondrial DNA control region sequences to the nucleus. Proc. Natl. Acad. Sci. USA 93, 15239-15243.

Sorenson, M. D., and Quinn, T. W. (1998) Numts: A challenge for avian systematics and population biology. Auk 115, $214-221$.

Tamura, K., Dudley, J., Nei, M., and Kumar, S. (2007) MEGA4: Molecular Evolutionary Genetics Analysis (MEGA) software version 4.0. Mol. Biol. Evol. 24, 1596-1599.

Tatarenkov, A., and Avise, J. C. (2007) Rapid concerted evolution in animal mitochondrial DNA. Proc. R. Soc. B 274, 1795-1798.

Walberg, M. W., and Clayton, D. A. (1981) Sequence and properties of the human $\mathrm{KB}$ cell and mouse $\mathrm{L}$ cell $\mathrm{D}$-loop regions of mitochondrial DNA. Nucleic Acids Res. 9, 5411-5421.

Wenink, P. W., Baker, A. J., and Tilanus, M. G. J. (1994) Mitochondrial control region sequences in two shorebird species, the Turnstone and the Dunlin, and their utility in population genetic studies. Mol. Biol. Evol. 11, 22-31.

Zhang, D. X., and Hewitt, G. M. (1996) Nuclear integrations: challenges for mitochondrial DNA markers. Trends Ecol. Evol. 11, 247-251.

Zimmer, E. A., Martin, S. L., Beverley, S. M., Kan, Y. W., and Wilson, A. C. (1980) Rapid duplication and loss of genes coding for the alpha chains of hemoglobin. Proc. Natl. Acad. Sci. USA 77, 7323-7327. 